\title{
COVID-19 Healthcare Worker Screening at Emergency Department, Hospital Wanita \& Kanak- Kanak Sabah, Malaysia from May-December 2020
}

\author{
${ }^{1,2}$ Mohamed Hakimi Abdul Kadir, ${ }^{1}$ Phee Kheng Cheah, ${ }^{2}$ Goh Geok Boon
}

1. Covid-19 Operations Room, Emergency and Trauma Department, Sabah Women and Children's Hospital, Kota Kinabalu, Sabah.

2. Pre-Hospital Unit, Emergency and Trauma Department, Sabah Women and Children's Hospital, Kota Kinabalu, Sabah.

\section{Preface}

Since the beginning of the pandemic we have recorded a total of 3,355 healthcare workers (HCW) screened at Emergency \& Trauma Department (ETD) of Sabah Women and Children's Hospital (HWKSS). This amounted to 8,823 swabs taken for HCWs from a total of 21,367 swabs taken in ETD during the same period. The average swab per HCW was $2.62 \mathrm{swab} /$ person.

Number of HCW and number of swabs taken

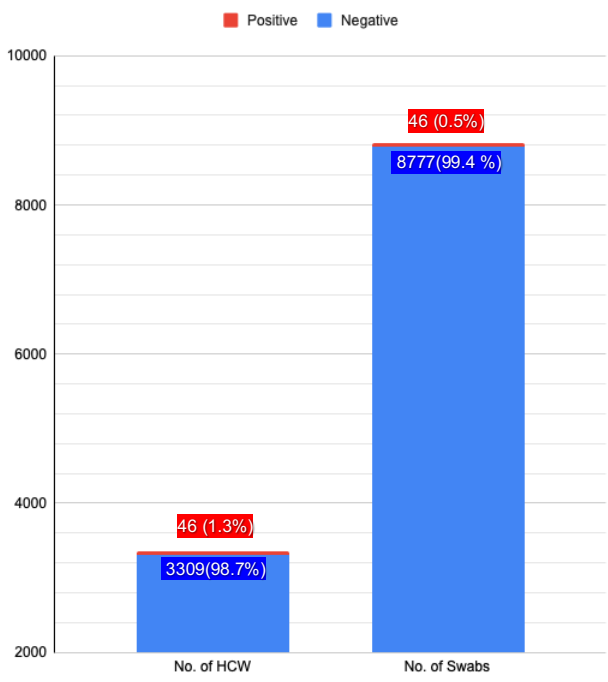

Chart 1 : First bar illustrates number of healthcare workers screened, while the second bar shows the number of swabs taken (including repeat swabs).

\section{HCW swabs taken per day at ETD HWKKS}

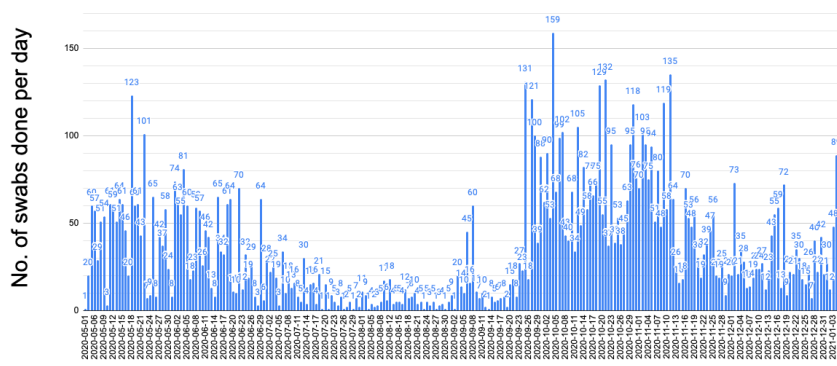

Dates

\section{Age Demographics}

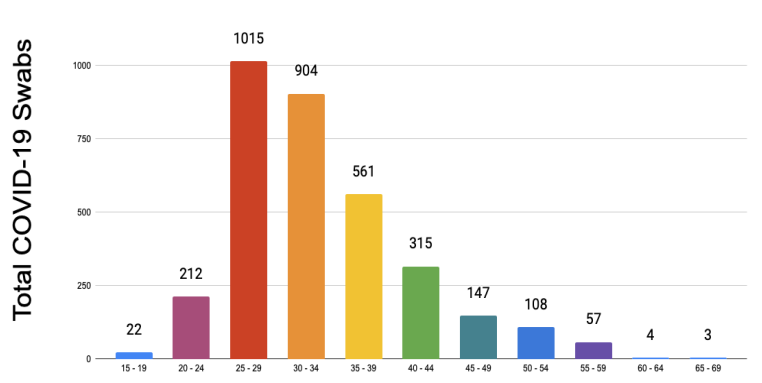

Age Group

\section{Screening Categories}

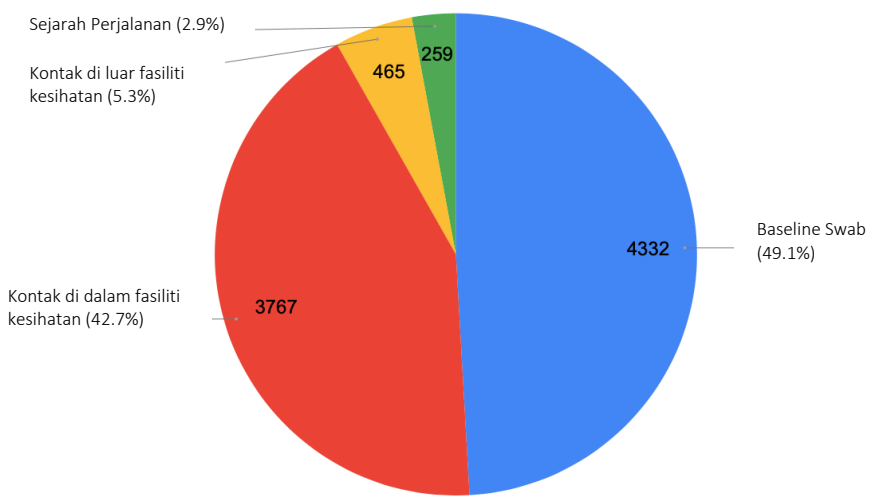

Chart 4 : Breakdown of indications for screening of HCWs in ETD HWKKS. (Baseline swab- done prior to reporting for duty or for rotational screening amongst HWKKS HCWs)

Correlation of symptoms and COVID-19 Swab Results

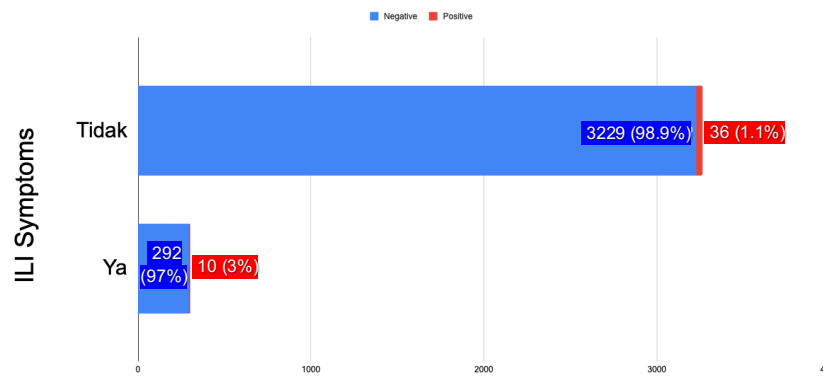

Healthcare Workers Screened

Chart 5 : Number of Healthcare Workers who are asymptomatic/symptomatic with relation to COVID-19 results.

\section{Limitations}

- $\quad$ Total staff in HWKKS is 2,093 but we found 3,355 individual registrations for $\mathrm{HCW}$ screening. This included $\mathrm{HCW}$ s from other facilities who opted for screening in HWKKS. This data also included relief staff from other facilities deployed to HWKKS during the height of the pandemic response. Support staff from our concession company (Sedafiat) security guards and other non-medical staff are also included in this database.

- This statistics excludes HCWs who had done their screening at their individual departments other than ETD.

- A total of 76 HWKKS HCWs were detected to have COVID19 during the data collection period. However, only 46 had their screening done in ETD.

\section{Corresponding Author}

Dr. Goh Geok Boon

Emergency \& Trauma Department

Sabah Women \& Children's Hospital

187, Sabah Karung Berkunci, 88996 Kota Kinabalu, Sabah wen.goh.520@gmail.com 